\title{
Prevalência e perfil dos pacientes que utilizam antipsicóticos em um hospital do sul do Brasil
}

\author{
Prevalence and profile of patients on antipsychotics in a hospital in southern Brazil
}

\author{
André Souza e Silva ${ }^{1}$, Fernanda Emy Inumaru ${ }^{1}$, Rafael Vidor ${ }^{1}$, André Luciano Manoel ${ }^{1}$, Thamy Dos Santos ${ }^{1}$, \\ Ana Carolina Carvalho', Daisson José Trevisol1, 2,3凶 \\ ${ }^{1}$ Faculdade de Medicina da Universidade do Sul de Santa Catarina. Tubarão, SC, Brasil. \\ 2 Programa de Pós Graduação em Ciências da Saúde da Universidade do Sul de Santa Catarina. Tubarão, SC, Brasil. \\ ${ }^{3}$ Centro de Pesquisas Clínicas do Hospital Nossa Senhora da Conceição. Tubarão, SC, Brasil.
}

\section{RESUMO}

Objetivos: Avaliar a prevalência e o perfil de pacientes hospitalizados que receberam prescrição de medicamentos antipsicóticos.

Métodos: Este foi um estudo de delineamento transversal que avaliou pacientes atendidos durante o ano de 2013 no Hospital Nossa Senhora da Conceição, em Tubarão, estado de Santa Catarina e que fizeram uso de medicamentos antipsicóticos. A coleta de dados aconteceu integralmente nos prontuários eletrônicos. Os pacientes foram avaliados de acordo com suas características sociodemográficas, tipo de antipsicótico utilizado e diagnóstico clínico pelo Código Internacional de Doenças (CID 10).

Resultados: De 125.670 pacientes atendidos no HNSC em 2013, 1.559 receberam algum medicamento antipsicótico. A média de idade desses pacientes foi de 50,34 anos ( $\mathrm{DP}=22,89)$. Não houve variação na prevalência de prescrição de antipsicóticos de acordo com o gênero e notou-se aumento nesta frequência de acordo com a idade dos pacientes. Os antipsicóticos mais prescritos foram os típicos; entre estes o haloperidol foi o mais utilizado, em 852 (54,7\%) pacientes. Entre os diagnósticos principais, os transtornos ansiosos foram os mais prevalentes, estando presentes em $131(6,3 \%)$ pacientes. Considerando somente diagnósticos de transtornos mentais ou comportamentais e incluindo os diagnósticos secundários, os transtornos ansiosos estiveram presentes em $233(14,9 \%)$ pacientes. Apenas $470(30,1 \%)$ pacientes que utilizaram antipsicóticos tinham, pelo menos, um diagnóstico de transtorno mental ou comportamental registrado.

Conclusões: Houve uma taxa de prevalência de $1,24 \%$ de uso de antipsicóticos nos pacientes do Hospital Nossa Senhora da Conceição, sendo haloperidol o antipsicótico mais prescrito. O diagnóstico de transtornos ansiosos foi o mais frequente entre os principais, porém grande parte dos pacientes que receberam antipsicóticos não tinham nenhum diagnóstico psiquiátrico, recebendo-os por outras causas.

DESCRITORES: antipsicóticos; haloperidol; transtornos mentais; ansiedade.

\section{ABSTRACT}

Aims: To evaluate the prevalence and the profile of inpatients prescribed antipsychotics.

Methods: This was a cross-sectional study which evaluated patients admitted to Hospital Nossa Senhora da Conceição (HNSC), in Tubarão, State of Santa Catarina, Brazil, in 2013, who were prescribed antipsychotic drugs. All the data were collected from electronic medical records. Patients were evaluated according to their sociodemographic characteristics, type of antipsychotic drug prescribed, and clinical diagnosis (ICD 10).

Results: Out of 125,670 patients admitted to HNSC in 2013, 1,559 received some kind of antipsychotic. The mean age of the patients was 50.34 years $(\mathrm{SD}=22.89)$. There was no variation in the prevalence of antipsychotic drug prescription between male and female patients, but the frequency of these prescriptions increased with age. Conventional antipsychotics were the most commonly prescribed ones, among which haloperidol was prescribed to $852(54.7 \%)$ patients. Among the major diagnoses, anxiety disorders were the most prevalent ones, detected in $131(6.3 \%)$ patients. Considering only the diagnoses of mental or behavioral disorders and including secondary diagnoses, anxiety disorders were observed in $233(14.9 \%)$ patients. A total of $470(30.1 \%)$ patients on antipsychotics had been diagnosed at least once with mental or behavioral disorders.

Conclusions: The prevalence of antipsychotic drug use was $1.24 \%$ among the inpatients, and haloperidol was the most widely prescribed drug. The diagnosis of anxiety disorders was the most frequent one, but most patients who received antipsychotics had not been diagnosed with any psychiatric disorder, and eventually received them for other reasons.

KEY WORDS: antipsychotics; haloperidol; mental disorders; anxiety. 
Abreviaturas: HNSC, Hospital Nossa Senhora da Conceição; OMS, Organização Mundial da Saúde; CID, Código Internacional de Doenças; DP, desvio padrão; DSM-IV, Diagnóstico e Estatística dos Transtornos Mentais, Quarta Edição.

\section{INTRODUÇÃO}

Segundo a Organização Mundial da Saúde (OMS), os medicamentos são substâncias fundamentais para a medicina, sendo essencial que sejam seguros, eficazes, de boa qualidade, e, principalmente, que sejam prescritos e utilizados de forma racional [1]. O consumo de medicamentos no Brasil tem aumentado nos últimos tempos em decorrência do mais fácil acesso e da grande variedade de produtos disponíveis no mercado [2]. Atualmente, mais da metade da população brasileira utiliza medicamentos, sendo que a prevalência aumenta conforme a idade dos indivíduos, além de ser maior em mulheres e pessoas de melhor nível socioeconômico $[2,3]$. Tem-se demonstrado que o maior percentual de uso de medicamentos concentra-se nos anti-inflamatórios e analgésicos [3].

Um grupo de fármacos bastante prescrito é o dos antipsicóticos, conhecidos por serem os medicamentos mais utilizados e de suma importância para o tratamento de esquizofrenia. Além de melhorar os sintomas dessa doença, esses fármacos são capazes de minimizar recorrências e diminuir o risco global de mortalidade [4-6]. Nos últimos anos surgiram novos medicamentos antipsicóticos, denominados de atípicos ou de segunda geração, que comprovadamente oferecem algumas vantagens sobre os chamados antipsicóticos típicos ou de primeira geração [4].

Os antipsicóticos típicos têm seu mecanismo de ação baseado no antagonismo de receptores $\mathrm{D}_{2}$ dopaminérgicos, com os quais se veiculam perfeitamente e se dissociam lentamente, gerando assim um melhor efeito contra sintomas psicóticos. Entretanto, essa característica faz com que o fármaco seja menos seguro, expondo seus usuários a efeitos colaterais, como discinesia tardia e reações extrapiramidais [4-7]. Já os antipsicóticos atípicos possuem maior afinidade por receptores serotoninérgicos $5-\mathrm{HT}_{2 \mathrm{~A}}$, e menor afinidade por receptores dopaminérgicos, explicando assim a menor capacidade de gerar os efeitos colaterais supracitados [8]. Apresentam vantagens como melhor ação sobre os sintomas negativos, menores índices de recaída e melhora no déficit cognitivo [4]. Em contrapartida a esses benefícios, alguns efeitos colaterais desses fármacos devem ser levados em conta, como hiperglicemia, ganho de peso e dislipidemia [9].

Os antipsicóticos são a classe de medicamentos mais prescrita em Centros de Atenção Psicossocial e a quarta classe prescrita em Ambulatórios de Saúde Mental [10]. O consumo desses fármacos psicoativos, ou seja, agem no sistema nervoso central, tem aumentado nas últimas décadas em muitos países ocidentais [11]. Segundo Gonçalves et al. [12], no ambiente extrahospitalar a prescrição dessa classe de medicamentos foi significativamente maior para homens. Entre os jovens foi comum a associação com benzodiazepínicos, tendo sido esse o tipo de associação medicamentosa, entre os fármacos psicoativos, mais frequente nessa faixa etária. Não parece existir influência de estado civil ou nível de escolaridade no uso de antipsicóticos nesse meio. O haloperidol, um antipsicótico típico, apesar de ser um dos mais antigos, esteve no topo da lista de prescrições [12].

O uso racional de medicamentos, segundo a OMS, é o conceito que diz que pacientes devem receber medicamentos apropriados para suas condições clínicas, em doses adequadas para suas necessidades, pelo menor tempo necessário e pelo menor custo possível. Estima-se que a utilização de mais da metade de todos os medicamentos prescritos no mundo, estão sendo feitos de forma errônea, gerando desperdícios e riscos para a saúde [1].

Os antipsicóticos estão indicados para tratamento de diversos transtornos mentais, entre eles a esquizofrenia, transtorno do humor bipolar, distúrbios psicóticos e hiperatividade, amenizando os consideráveis transtornos que algumas doenças mentais trazem ao seu portador. Entretanto, se não prescritos e usados de forma correta, poderão gerar uma gama expressiva de efeitos colaterais, como os já citados $[4,9,13]$.

É importante traçar o perfil do uso de antipsicóticos em pacientes hospitalizados, para que seja possível comparar e confrontar os dados de literatura, buscando informações que possam auxiliar a melhor racionalizar o uso desse tipo de medicamento. Assim, o objetivo desse estudo foi avaliar a prevalência e o perfil sociodemográfico e clínico dos pacientes que utilizaram antipsicóticos no Hospital Nossa Senhora da Conceição (HNSC) de Tubarão, Santa Catarina, no ano de 2013.

\section{MÉTODOS}

Foi realizado um estudo epidemiológico exploratório com delineamento transversal. $\mathrm{O}$ ambiente da pesquisa foi o HNSC, hospital geral que fica localizado 
em Tubarão, estado de Santa Catarina, cidade que possui 92.569 habitantes. O HNSC é o maior hospital em número de leitos do estado de Santa Catarina.

O HNSC utiliza a plataforma eletrônica de gestão de serviços em saúde Tasy ${ }^{\circledR}$ (Wheb Sistemas, Philips Clinical Informatics, Blumenau, SC). Por meio de pesquisa nos prontuários eletrônicos, foram localizados os pacientes que utilizaram medicamentos antipsicóticos durante a hospitalização, no ano de 2013. Foram excluídos do estudo os pacientes para os quais foi prescrito sulpirida, por ser um fármaco amplamente utilizado apenas pelo seu efeito colateral hiperprolactinêmico, como estimulante da produção de leite para as mães com dificuldade na amamentação. Muitas vezes, inclusive, esse medicamento é prescrito no prontuário do recém-nascido para uso materno.

O estudo foi aprovado pelo Comitê de Ética em Pesquisa da Unisul sob o número 491.084 e atendeu a todos os requisitos da Resolução 466 de 2012 do Conselho Nacional de Saúde. Os dados foram coletados após a autorização de acesso aos prontuários eletrônicos pela direção do HNSC, sendo mantida em sigilo a identificação dos pacientes. Em virtude de se tratar de uma coleta de dados em prontuário, foi dispensada a assinatura do termo de consentimento.

Para a coleta de dados foi utilizado um roteiro contendo as variáveis de interesse para a pesquisa: idade, gênero, escolaridade (em anos de estudo), situação conjugal, cor da pele, ocupação, medicamento antipsicótico utilizado e diagnóstico conforme o Código Internacional de Doenças (CID) em sua versão CID 10. Os diagnósticos e seus correspondentes CID foram determinados pelo médico assistente no momento do atendimento ao paciente. Tal profissional geralmente é um médico generalista, que inicia a conduta e, se julgar necessário, transfere o doente aos cuidados de um médico especialista. Este, então, prossegue com os cuidados ao paciente durante a internação. Portanto, o diagnóstico e a classificação CID do atendimento foram na maioria das vezes realizados por um médico generalista ou especialista em área médica que na maior parte das vezes não era a psiquiatria.

Os códigos coletados para a pesquisa foram os relacionados a diagnósticos de transtornos mentais e comportamentais, registrados durante qualquer atendimento do paciente no HNSC no ano de 2013.

Os dados foram inseridos no software Epidata versão 3.1 e a análise estatística foi feita com auxílio do IBM SPSS Statistics V21.0. As variáveis quantitativas foram descritas com medidas de tendência central e dispersão, enquanto para a exposição das variáveis qualitativas utilizaram-se números absolutos e proporções. Para verificar associação entre as variáveis de interesse, foi aplicado o Teste do qui-quadrado para comparações bivariadas e Teste $t$ de Student para comparação de médias. O intervalo de confiança pré-estabelecido foi de $95 \%$ com erro $\alpha$ de $5 \%$. Foram considerados estatisticamente significativas associações que apresentaram valores de $\mathrm{p}<0,05$.

\section{RESULTADOS}

Do total de 125.670 pacientes atendidos no HNSC em 2013, 1.559 (1,24\%) houve prescrição de algum medicamento antipsicótico intrahospitalar. Esses 1.559 pacientes tiveram 1.813 atendimentos hospitalares, incluindo hospitalizações e consultas, com uma média de 1,16 atendimentos por paciente durante o ano de 2013.

A média de idade dos pacientes foi de 50,34 anos $(\mathrm{DP}=22,89)$. Não houve diferença entre o uso de antipsicóticos relacionado ao gênero, enquanto a prevalência aumentou progressivamente em relação à idade dos pacientes. Os resultados em relação ao perfil sociodemográfico desses pacientes estão apresentados na Tabela 1.

Tabela 1. Perfil sociodemográfico dos pacientes que utilizaram antipsicóticos no ano de 2013 no Hospital Nossa Senhora da Conceição, Tubarão, Santa Catarina.

\begin{tabular}{|c|c|c|c|}
\hline Características & $\begin{array}{c}n \\
(1.559)\end{array}$ & $\begin{array}{c}\% \\
(100 \%)\end{array}$ & $\begin{array}{c}\text { Intervalo } \\
\text { de confiança } \\
95 \%\end{array}$ \\
\hline $\begin{array}{l}\text { Sexo } \\
\text { Masculino } \\
\text { Feminino }\end{array}$ & $\begin{array}{l}734 \\
825\end{array}$ & $\begin{array}{l}47,1 \\
52,9\end{array}$ & $\begin{array}{l}44,6-49,5 \\
50,5-55,4\end{array}$ \\
\hline $\begin{array}{l}\text { Idade em anos } \\
0-19 \\
20-39 \\
40-59 \\
60 \text { ou mais }\end{array}$ & $\begin{array}{l}121 \\
430 \\
456 \\
552\end{array}$ & $\begin{array}{l}7,8 \\
27,6 \\
29,2 \\
35,4\end{array}$ & $\begin{array}{c}6,5-9,2 \\
25,3-29,9 \\
27,0-31,7 \\
32,9-37,7\end{array}$ \\
\hline $\begin{array}{l}\text { Escolaridade }(\mathrm{N}=1349) \\
\text { Não alfabetizado/analfabeto } \\
\text { Até } 8 \text { anos de estudo } \\
\text { Mais de } 8 \text { anos de estudo }\end{array}$ & $\begin{array}{l}132 \\
636 \\
574\end{array}$ & $\begin{array}{c}9,8 \\
47,4 \\
42,8\end{array}$ & $\begin{array}{l}8,3-11,5 \\
44,7-50,1 \\
40,2-45,5\end{array}$ \\
\hline $\begin{array}{l}\text { Estado Civil }(\mathrm{N}=1536) \\
\text { Com companheiro } \\
\text { Sem companheiro }\end{array}$ & $\begin{array}{l}837 \\
699\end{array}$ & $\begin{array}{l}54,5 \\
45,5\end{array}$ & $\begin{array}{l}52,1-57,1 \\
42,9-47,9\end{array}$ \\
\hline $\begin{array}{l}\text { Cor da pele } \\
\text { Branca } \\
\text { Não branca }\end{array}$ & $\begin{array}{c}1.451 \\
108\end{array}$ & $\begin{array}{c}93,1 \\
6,9\end{array}$ & $\begin{array}{c}93,1 \\
6,9\end{array}$ \\
\hline $\begin{array}{l}\text { Ocupação }(\mathrm{N}=1330) \\
\text { Estudante/menor } \\
\text { Desempregado/do lar } \\
\text { Economicamente ativo } \\
\text { Aposentado/pensionista }\end{array}$ & $\begin{array}{l}103 \\
143 \\
586 \\
453\end{array}$ & $\begin{array}{c}8 \\
11,1 \\
45,6 \\
35,3\end{array}$ & $\begin{array}{c}6,5-9,5 \\
9,4-12,9 \\
42,9-48,2 \\
32,8-38,1\end{array}$ \\
\hline
\end{tabular}


Quanto aos antipsicóticos utilizados, os típicos foram o haloperidol, a clorpromazina e a levomepromazina; o antipsicótico atípico foi a risperidona; e também foi identificado o uso da associação citrato de fentanila/ haloperidol. Observou-se que houve preferência pelo uso do haloperidol e pelos antipsicóticos típicos em geral (Tabela 2).

Tabela 2. Prevalência de utilização de antipsicóticos típicos e atípicos em pacientes atendidos no Hospital Nossa Senhora da Conceição, Tubarão, Santa Catarina, no ano de 2013.

\begin{tabular}{lccc}
\hline \multicolumn{1}{|c}{ Antipsicóticos utilizados } & $\mathbf{n}$ & $\mathbf{\%}$ & $\begin{array}{c}\text { Intervalo de } \\
\text { confiança 95\% }\end{array}$ \\
\hline Antipsicóticos Tipicos & & & \\
Haloperidol & 852 & 54,7 & $52,3-57,2$ \\
Clorpromazina & 527 & 33,8 & $31,6-36,3$ \\
$\quad$ Levomepromazina & 270 & 17,3 & $15,6-19,2$ \\
Antipsicóticos Atípicos & & & \\
$\quad$ Risperidona & 268 & 17,2 & $15,3-19,3$ \\
$\quad$ Fentanil + Droperidol & 84 & 5,4 & $4,3-6,6$ \\
\hline
\end{tabular}

Observação: alguns pacientes fizeram uso de mais de um medicamento.
Os CID registrados na hospitalização dos pacientes no HNSC no ano de 2013 estão listados por ordem decrescente de frequência na Tabela 3. Os CID que possuíam três números foram simplificados para dois números. Destaque para o CID F41, que representa transtornos ansiosos e foi o mais frequente, sendo citado 131 vezes $(6,3 \%)$.

A Tabela 4 apresenta os CID levando em conta apenas os diagnósticos de distúrbios psiquiátricos que foram registrados durante a hospitalização.

Verificou-se também que apenas $470(30,1 \%)$ pacientes que utilizaram antipsicóticos tinham, pelo menos, um CID referente a diagnóstico psiquiátrico registrado durante seu atendimento no HNSC, seja hospitalização ou consulta. Assim, grande parte dos pacientes que receberam antipsicóticos no ano de 2013 no HNSC não tinham nenhum diagnóstico psiquiátrico, recebendo-os por outras causas.

Quando comparados os pacientes que utilizaram antipsicótico e que tiveram diagnóstico de algum transtorno mental ou comportamental com os que não tiveram esses diagnósticos, verificou-se que houve
Tabela 3. Principais diagnósticos, conforme o Código Internacional de Doenças versão 10 (CID 10) dos pacientes que receberam antipsicóticos no ano de 2013 no Hospital Nossa Senhora da Conceição, Tubarão, Santa Catarina.

\begin{tabular}{lcc}
\hline \multicolumn{1}{c}{ Diagnóstico do atendimento (CID 10) } & $\mathbf{n}$ & $\%$ \\
\hline Outros transtornos ansiosos (F41) & 131 & 6,3 \\
\hline Dor abdominal e pélvica (R10) & 67 & 3,2 \\
\hline Cefaleia (R51) & 65 & 3,1 \\
\hline Hipertensão materna não especificada* (O16) & 64 & 3,1 \\
\hline Pneumonia bacteriana não classificada em outra parte (J15) & 57 & 2,7 \\
\hline Anormalidades da respiração (R06) & 55 & 2,6 \\
\hline Episódios depressivos (F32) & 46 & 2,2 \\
\hline Outros transtornos do trato urinário (N39) & 43 & 2,1 \\
\hline Angina pectoris (I20) & 34 & 1,6 \\
\hline Pneumonia por microorganismo não especificado (J18) & 33 & 1,6 \\
\hline Traumatismo intracraniano (S06) & 32 & 1,5 \\
\hline Fratura do fêmur (S72) & 31 & 1,5 \\
\hline Insuficiência cardíaca (I50) & 30 & 1,4 \\
\hline Acidente vascular cerebral não especificado (I64) & 30 & 1,4 \\
\hline Transtornos mentais e comportamentais devidos ao uso de álcool (F10) & 29 & 1,4 \\
\hline Enxaqueca (G43) & 27 & 1,3 \\
\hline Dor de garganta e no peito (R07) & 27 & 1,3 \\
\hline Transtorno afetivo bipolar (F31) & 26 & 1,2 \\
\hline Hipertensão essencial (I10) & 26 & 1,2 \\
\hline Náusea e vômitos (R11) & 26 & 1,2 \\
\hline Infarto agudo do miocárdio (I21) & 25 & 1,2 \\
\hline Transtornos psicóticos agudos e transitórios (F23) & 24 & 1,1 \\
\hline Epilepsia (G40) & 21 & 1 \\
\hline Outros transtornos articulares não classificados em outra parte (M25) & 20 & 1 \\
\hline Fratura da perna, incluindo tornozelo (S82) & 20 & 1 \\
\hline Outros & 1099 & 52,6 \\
\hline & & \\
\hline
\end{tabular}

* Hipertensão em puérpera. 
Tabela 4. Frequências entre os diagnósticos psiquiátricos dos pacientes atendidos no Hospital Nossa Senhora da Conceição, Tubarão, Santa Catarina, no ano de 2013, conforme o Código Internacional de Doenças versão 10 (CID 10).

\begin{tabular}{lcc}
\hline \multicolumn{1}{c}{ Diagnóstico (CID 10) } & n & \% \\
\hline Outros transtornos ansiosos (F41) & 233 & 14,9 \\
Episódios depressivos (F32) & 107 & 14,8 \\
Transtornos mentais e comportamentais devidos ao uso de álcool (F10) & 56 & 7,7 \\
Transtorno afetivo bipolar (F31) & 446 & 6,3 \\
Transtornos psicóticos agudos e transitórios (F23) & 32 & 4,4 \\
Transtorno depressivo recorrente (F33) & 28 & 3,9 \\
Transtornos somatoformes (F45) & 23 & 3,2 \\
Esquizofrenia (F20) & 19 & 2,6 \\
Outros transtornos mentais devidos a lesão e disfunção cerebral e a doença física (F06) & 18 & 2,5 \\
Transtornos mentais e comportamentais devidos ao uso da cocaína (F14) & 17 & 2,3 \\
Transtornos específicos da personalidade (F60) & 17 & 2,3 \\
Psicose não orgânica não especificada (F29) & 16 & 2,2 \\
\hline
\end{tabular}

Estão apresentados nesta tabela apenas os diagnósticos com frequência maior que 2\%. associação entre ter recebido um CID de doença mental ou comportamental e ser mulher $(p<0,001)$, ter cor de pele não-branca $(\mathrm{p}=0,002)$, ter mais de 8 anos de estudo $(p<0,001)$ e ter entre $40-59$ anos $(p<0,001)$.

\section{DISCUSSÃO}

A prevalência de uso de medicamentos antipsicóticos em pacientes hospitalizados no HNSC foi de $1,24 \%$, não havendo diferença significativa quando comparados homens e mulheres. O uso de antipsicóticos aumentou progressivamente com o aumento da idade dos pacientes atendidos.

Cabe ressaltar que os antipsicóticos foram utilizados para tratamento ou alívio de sintomas em uma gama bastante heterogênea de situações clínicas e em geral, a indicação dos antipsicóticos foi adequada, segundo parâmetros da literatura [13].

O estudo de Machado et al. [14], realizado em Maringá, identificou uma predominância de prescrição de antipsicóticos para mulheres e para pacientes com idade entre 21 e 50 anos. Quando comparado com o presente estudo, houve semelhança para o sexo e uma ligeira diferença com relação a faixa etária (entre 40 e 59 anos). Além disso, observou-se que o antipsicótico mais utilizado pelos pacientes de ambos estudos foi o haloperidol, um antipsicótico típico, dos mais antigos do mercado e de melhor relação custo-benefício [14].

Talvez o uso de um antipsicótico típico como o medicamento mais amplamente utilizado neste estudo se justifique pelo fato de que ainda há controvérsias em relação à indicação de antipsicóticos atípicos para agitação psicomotora, um quadro muito comum em hospitais gerais, que leva à necessidade do uso desse tipo de medicamento. Vale ressaltar que os antipsicóticos atípicos podem levar a uma gama de efeitos colaterais indesejados quando utilizados para algumas causas de agitação psicomotora, além de se encontrarem menos disponíveis atualmente [15-17].

Estudo realizado em uma clínica de hospitalização psiquiátrica chilena, com pacientes internados que utilizaram antipsicóticos, observou que na maioria dos casos houve associação de uso de um antipsicótico típico com um atípico. A média de idade dos pacientes daquele estudo foi de 34 anos, sendo que 50,9\% eram mulheres e $54 \%$ tinham alguma comorbidade psiquiátrica. As comorbidades psiquiátricas mais frequentes foram transtorno de personalidade, transtorno bipolar, farmacodependência e depressão [18].

No presente estudo a média de idade foi maior do que no estudo chileno, entretanto houve semelhança de frequência de prescrição em relação ao sexo (52,9\% mulheres). Além disso na amostra presente foram utilizados principalmente antipsicóticos típicos, sem associação com atípicos $(82,8 \%$ dos casos $)$. O número de comorbidades psiquiátricas foi menor, provavelmente porque o estudo chileno foi realizado em uma clínica de internação psiquiátrica e teve como critério de exclusão o uso esporádico de haloperidol intramuscular. As comorbidades psiquiátricas mais frequentes dos pacientes dos dois estudos foram semelhantes em partes, porém essa comparação deve ser analisada com cuidado, uma vez que o presente estudo utilizou o CID 10, enquanto o estudo chileno utilizou o Manual de Diagnóstico e Estatística dos Transtornos Mentais, Quarta Edição (DSM-IV), publicado pela Associação Psiquiátrica Americana [18].

O estudo de Shirama et al. [19] avaliou o perfil de pacientes hospitalizados que utilizaram qualquer classe de psicofármacos e mostrou maior prevalência de uso de 
psicofármacos por mulheres. Além disso, houve maior prevalência de uso destes medicamentos em pacientes com menos de oito anos de escolaridade, com mais de 51 anos de idade e sem companheiros. Já em relação ao número de prescrições, os antipsicóticos ficaram atrás apenas dos benzodiazepínicos e antidepressivos [19]. Esses resultados convergem com os dados encontrados no presente estudo, com exceção da situação conjugal [19].

Em estudo realizado em uma unidade de hospitalização psiquiátrica em Zaragoza, Espanha, observou-se que o maior número de prescrições de antipsicóticos eram destinados a homens, não ativos, com menos de seis anos de estudo e solteiros. Os diagnósticos mais encontrados foram esquizofrenia, transtorno bipolar e transtorno psicótico agudo. O antipsicótico mais utilizado em monoterapia foi a risperidona e, quando em associação com outro antipsicótico, o escolhido foi o haloperidol. No geral, os antipsicóticos atípicos predominaram. Com exceção da escolaridade, os dados sociodemográficos foram divergentes dos resultados do presente estudo. A divergência de resultados provavelmente se deu pelo fato do estudo espanhol ter sido realizado em uma unidade de hospitalização psiquiátrica [20].

É possível observar que os diversos estudos que avaliaram o uso de antipsicóticos em regime intrahospitalar obtiveram resultados discrepantes entre si. Contudo, isso não necessariamente significa que a prática do uso desse tipo de medicação nesses hospitais aconteceu de maneira equivocada. A disparidade pode representar uma diferença de preferências entre medicamentos e uma desigualdade entre as populações atendidas, além de diferentes metodologias de estudo utilizadas $[14,18,20]$.

Um grande número de pacientes recebeu a prescrição de antipsicóticos visando ao tratamento da doença que motivou o atendimento hospitalar, porém muitos podem ter recebido tal prescrição visando apenas ao alívio de sintomas, como agitação psicomotora. Sabe-se que na prática médica muitas vezes os antipsicóticos são usados apenas para o controle de uma agitação psicomotora momentânea [21], o que é muito comum em hospitais gerais como o HNSC. Isso poderia, em tese, explicar a notável quantidade de prescrições nas quais não há um motivo aparente ou um diagnóstico clínico definitivo que demande o seu uso. Porém isso não pode ser afirmado com certeza com base nos dados disponíveis.

Alguns efeitos colaterais que podem ocorrer com o uso dos antipsicóticos são discinesia tardia, acatisia, parkinsonismo, síndrome neuroléptica maligna, hiperglicemia, ganho de peso, dislipidemia. Os antipsicóticos são medicamentos muito úteis na prática médica, mas devem ser usados com a devida cautela [4,22].

Como limitação deste estudo, podemos destacar que não foi possível comparar o perfil dos pacientes que utilizaram antipsicóticos com os que não utilizaram. Além disso, por ser um estudo realizado em prontuários eletrônicos, pode ter havido perda de dados ou dados registrados de maneira incorreta. Cabe ressaltar uma limitação importante do estudo, que é o fato de que os diagnósticos e a escolha do CID foram feitos por médicos generalistas e médicos especialistas em diversas áreas, e não apenas por psiquiatras, o que pode gerar dúvida nos diagnósticos realizados.

Mesmo considerando essas limitações, os dados deste estudo podem ser importantes para os profissionais que trabalham na rede hospitalar, pois revelam um perfil epidemiológico de pacientes que, por algum motivo, necessitaram do uso de antipsicóticos. Conclui-se, assim, que a prevalência do uso de antipsicóticos nos pacientes que deram entrada no HNSC no ano de 2013 foi de $1,24 \%$, sendo que em mais de $90 \%$ desses pacientes os antipsicóticos prescritos foram os típicos. Não houve diferença significativa na prevalência do uso de antipsicóticos quanto ao gênero, mas esta aumentou progressivamente com o aumento da idade dos pacientes. Considerando apenas os pacientes que receberam antipsicóticos, os diagnósticos de transtornos mentais e comportamentais foram mais frequentes em mulheres, em não-brancos, em pacientes com maior escolaridade e nos pacientes com idade entre $40 \mathrm{e}$ 59 anos. O diagnóstico de transtornos ansiosos foi o mais frequente entre os principais, porém grande parte dos pacientes que receberam antipsicóticos não tinham nenhum diagnóstico psiquiátrico, recebendo-os por outras causas.

\section{REFERÊNCIAS}

1. World Health Organization. WHO model list of Essential Medicines 19th Ed [Internet]. Geneva; 2015. [cited 2015 May]. Available from: http:// www.who.int/entity/selection_medicines/committees/expert/20/EML_2015_FINAL_amended_AUG2015.pdf?ua=1

2. Costa KS, Barros MBA, Franscisco PMSB, César CLG, Goldbaum M, Carandina L. Utilização de medicamentos e fatores associados: um estudo de base populacional no Município de Campinas, São Paulo, Brasil. Cad Saúde Pública. 2011;27(4):649-58. http://dx.doi.org/10.1590/ S0102-311X2011000400004 
3. Lambrey S, Falissard B, Martin-Barrero M, Bonnefoy C, Quilici G, Rosier A, et al. Effectiveness of clozapine for the treatment of aggression in an adolescent with autistic disorder. J Child Adolesc Psychopharmacol. 2010;20(1):79-80. http://dx.doi.org/10.1089/cap.2009.0057

4. Faculdade de Medicina da Universidade de São Paulo. Interações medicamentosas em psiquiatria [Internet]. São Paulo; 2014. [cited 2015 May]. Available from: http://www.ipqhc.org.br/pdfs/Psico.pdf

5. Page VJ, Ely EW, Gates S, Zhao XB, Alce T, Shintani A, et al. Effect of intravenous haloperidol on the duration of delirium and coma in critically ill patients (Hope-ICU): a randomised, double-blind, placebo-controlled trial. Lancet Respir Med. 2013;1(7):515-23. http://dx.doi. org/10.1016/S2213-2600(13)70166-8

6. Komossa K, Rummel-Kluge C, Schwarz S, Schmid F, Hunger H, Kissling W, et al. Risperidone versus other atypical antipsychotics for schizophrenia. Cochrane Database Syst Rev. 2011;19;(1):CD006626. http://dx.doi.org/10.1002/14651858.cd006626.pub2

7. Divac N, Prostran M, Jakovcevski, Cerovac N. Second-generation antipsychotics and extrapyramidal adverse effects. Biomed Res Int 2014;6(5)63-70. http://dx.doi.org/10.1093/schbul/sbq042

8. Meltzer HY, Matsubara S, Lee JC. Classification of typical and atypical antipsychotic drugs on the basis of dopamine D-1, D-2 and serotonin2 pKi values. J Pharmacol Exp Ther. 1989;251(1):238-46.

9. Khasawneh FT, Shankar GS. Minimizing cardiovascular adverse effects of atypical antipsychotic drugs in patients with schizophrenia. Cardiol Res Pract. 2014;2(7)30-60. http://dx.doi.org/10.1155/2014/273060

10. Pereira MO, Souza JM, Costa AM, Vargas D, Oliveira MAF, Moura WN. Perfil dos usuários de serviços de Saúde Mental do município de Lorena - São Paulo. Acta Paul Enferm. 2012;25(1):48-54. http://dx.doi.org/10.1590/S0103-21002012000100009

11. Pincus HA, Tanielian TL, Marcus SC, Olfson M, Zarin DA, Thompson J, et al. Prescribing trends in psychotropic medications: primary care, psychiatry, and other medical specialties. JAMA. 1998;279(7):526-31. http://dx.doi.org/10.1001/jama.279.7.526

12. Gonçalves DP, Silva IV, Rangel LB, Rezende LC. Prescription of psychoactive drugs in patients attended by the SUS at Manhuaçu - MG (Brazil). Pharmacy Practice. 2011;9(4):200-6. http://dx.doi.org/10.4321/S1886-36552011000400004

13. Agency for Healthcare Research and Quality (US). Future Research Needs for First- and Second-Generation Antipsychotics for Children and Young Adults [Internet]. Rockville; 2012 [cited 2015 May]. Available from: http://www.ncbi.nlm.nih.gov/books/NBK84656/

14. Machado FB, Gomes MF, Junior SEM, Mella EAC. Determinação dos antipsicóticos utilizados no hospital municipal de Maringá - Paraná. Semina Cienc Biol Saúde. 2009;30(1):77-82.

15. Powney MJ1, Adams CE, Jones H. Haloperidol for psychosis-induced aggression or agitation (rapid tranquillisation). Cochrane Database Syst Rev. 2012; 14;11:CD009377.

16. Diógenes AR, Rodrigues FC, Xavier CA, Guerra GC, Alves MSCF, Ferreira AA. Análise da relação custo e consumo dos antipsicóticos típicos e atípicos: implicações para a saúde pública. Rev Ciênc Méd Biol. 2007;6(3):275-81.

17. Horn M, Vaiva G, Dumais A. Drug management of agitation in emergency departments: theoretical recommendations and studies of practices. Presse Med. 2015 Jan;44(1):20-6. http://dx.doi.org/10.1016/j.lpm.2014.04.027

18. Nieto RR, Silva HI. Prescripción de antipsicóticos atípicos en pacientes hospitalizados de la Clínica Psiquiátrica Universitaria. Rev Chil Neuropsiquiat. 2008;46(1):16-24. http://dx.doi.org/10.4067/S0717-92272008000100003

19. Shirama FH, Miasso AI. Consumo de psicofármacos por pacientes de clínicas médica e cirúrgica de um hospital geral. Rev Latino-Am Enfermagem. 2013;21(4):1-8.

20. Arilla JA, Fernández ME, Vi-uales E, Lozano I. Politerapia antipsicótica en una unidad de hospitalización psiquiátrica. Rev Psiquiatr Salud Ment (Barc). 2010;3(3):90-6. http://dx.doi.org/10.1016/j.rpsm.2010.04.001

21. Baldaçara L, Sanches M, Cordeiro DC, Jackowski AP. Tranquilização rápida para pacientes agitados nos serviços de emergência psiquiátrica: um ensaio clínico randomisado de olanzapina, ziprasidona, haloperidol mais prometazina, haloperidol mais midazolam e haloperidol em monoterapia. Rev Bras Psiquiatr. 2011;33(1):30-9. http://dx.doi.org/10.1590/S1516-44462011000100008

22. Young SL, Taylor M, Lawrie SM. "First do no harm." A systematic review of the prevalence and management of antipsychotic adverse effects J Psychopharmacol. 2015;29(4):353-62. http://dx.doi.org/10.1177/0269881114562090 